\title{
The Possible Role of Elastic Properties of the Brain and Optic Nerve Sheath in the Development of Spaceflight-Associated Neuro-Ocular Syndrome
}

W e read with great interest the article recently published in the American Journal of Neuroradiology by Roberts et al, ${ }^{1}$ and we appreciate the opportunity to make a comment.

The authors retrospectively analyzed brain MR imaging scans of National Aeronautics and Space Administration astronauts to quantify pre- to postflight changes in brain structure. ${ }^{1}$ Long-duration spaceflights aboard the International Space Station, but not short-duration Space Shuttle flights, resulted in a significant increase in the percentage of total ventricular volume change $(10.7 \%$ versus $0 \%, P<.001, n=12$ versus $n=7)$. The percentage of total ventricular volume change was significantly associated with the mission duration $(r=0.72$, $P=.001, n=19)$ but negatively associated with age $(r=$ $-0.48, P=.048, n=19)$. The authors also examined post hoc the extent to which pre- to postflight structural brain changes were associated with the development of spaceflight-associated neuro-ocular syndrome (SANS). They hypothesized that ventricular enlargement postflight would correlate with the development of SANS. However, of the 12 long-duration astronauts, the 4 astronauts with ophthalmologic changes had a smaller change in the percentage of total ventricular volume than those without SANS ( $12.8 \%$ versus $6.5 \%, P=.02, n=8$ versus $n=4$ ). This preliminary result suggested that ventricular enlargement, as seen in astronauts without SANS, may not be a characteristic feature of SANS. ${ }^{1}$ With regard to this apparently surprising finding, we would like to present an additional viewpoint.

In a recent article, Van Ombergen et $\mathrm{al}^{2}$ found an increased ventricular CSF volume after long-duration spaceflights in supratentorial ventricular structures (ie, lateral and the third ventricles) and further hypothesized that the impact on the supratentorial regions is an indicator of the structural compliance and CSF reserve capacity of these ventricles acting as buffers in healthy adults. According to the authors, this might be considered a preliminary coping mechanism in space, which acts as an intermediate overflow zone for the reduced CSF resorption. They further suggested a potential link between

http://dx.doi.org/10.3174/ajnr.A6430 the brain ventricular and CSF changes and symptoms of SANS, in the sense that a larger compensatory reserve capacity in the CSF space (eg, by a greater compliance and elasticity of the ventricles) may protect against ophthalmic changes in space travelers by delaying CSF pressure buildup in the retro-orbital space. Wostyn and De Deyn ${ }^{3,4}$ had previously proposed a similar compensatory mechanism at the level of the optic nerve sheath. They hypothesized that astronauts with less compliant optic nerve sheaths may be more likely to develop optic disc edema, given that a greater degree of optic nerve sheath rigidity that prevents further expansion may result in more CSF pressure increase in the subarachnoid space surrounding the optic nerve. According to these 2 hypotheses, microgravity-induced ocular changes may be partially determined by the elastic properties of the brain ventricles and optic nerve sheath. Thus, the entire CSF compartment, including the dural bulges around the cranial nerves, may act as a buffer zone against the development of SANS. This view is consistent with the smaller change in the percentage of total ventricular volume experienced by astronauts with SANS, as observed in the present study by Roberts et al. ${ }^{1}$

Disclosures: Peter Wostyn-UNRELATED: Patents (Planned, Pending or Issued): Peter Wostyn is the inventor of a pending patent application pertaining to biomarkers for spaceflight-associated neuro-ocular syndrome.

\section{REFERENCES}

1. Roberts DR, Asemani D, Nietert PJ, et al. Prolonged microgravity affects human brain structure and function. AJNR Am J Neuroradiol 2019;40:1878-85 CrossRef Medline

2. Van Ombergen A, Jillings $S$, Jeurissen B, et al. Brain ventricular volume changes induced by long-duration spaceflight. Proc Natl Acad Sci USA 2019;116:10531-36 CrossRef Medline

3. Wostyn P, De Deyn PP. Intracranial pressure-induced optic nerve sheath response as a predictive biomarker for optic disc edema in astronauts. Biomark Med 2017;11:1003-08 CrossRef Medline

4. Wostyn P, De Deyn PP. Optic nerve sheath distention as a protective mechanism against the visual impairment and intracranial pressure syndrome in astronauts. Invest Ophthalmol Vis Sci 2017;58:4601-02 CrossRef Medline 
(D) Postyn

Department of Psychiatry

PC Sint-Amandus

Beernem, Belgium

(D) T.H. Mader

Colonel, US Army (retired), Moab, Utah

DC.R. Gibson

Coastal Eye Associates Webster, Texas

KBR, Houston, Texas

(D)F.L. Wuyts

Lab for Equilibrium Investigations and Aerospace

University of Antwerp Antwerp, Belgium

(D) A. Van Ombergen

Lab for Equilibrium Investigations and Aerospace Department of Translational Neurosciences

University of Antwerp Antwerp, Belgium
(1) P. zu Eulenburg German Center for Vertigo and Balance Disorders Faculty of Medicine Ludwig-Maximilians-University Munich Munich, Germany

(D)P.P. De Deyn

Department of Biomedical Sciences Laboratory of Neurochemistry and Behavior Institute Born-Bunge University of Antwerp Antwerp, Belgium Department of Neurology and Alzheimer Research Center University of Groningen and University Medical Center Groningen Groningen, the Netherlands Department of Neurology and Memory Clinic Middelheim General Hospital Antwerp, Belgium 